Manfred Redelfs

Investigative Reporting in den USA 
Studien zur Kommunikationswissenschaft Band 21 
Manfred Redelfs

\section{Investigative Reporting in den USA}

Strukturen eines Journalismus

der Machtkontrolle

Westdeutscher Verlag 
Die Deutsche Bibliothek - CIP-Einheitsaufnahme

\section{Redelfs, Manfred:}

Investigative reporting in den USA: Strukturen eines Journalismus der Machtkontrolle / Manfred Redelfs. Opladen: Westdt. Verl., 1996

(Studien zur Kommunikationswissenschaft; Bd. 21)

NE: GT

Alle Rechte vorbehalten

(C) 1996 Westdeutscher Verlag GmbH, Opladen

Der Westdeutsche Verlag ist ein Unternehmen der Bertelsmann Fachinformation.

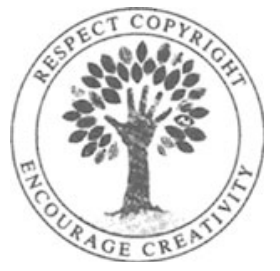

Das Werk einschließlich aller seiner Teile ist urheberrechtlich geschützt. Jede Verwertung außerhalb der engen Grenzen des Urheberrechtsgesetzes ist ohne Zustimmung des Verlags unzulässig und strafbar. Das gilt insbesondere für Vervielfältigungen, Übersetzungen, Mikroverfilmungen und die Einspeicherung und Verarbeitung in elektronischen Systemen.

Umschlaggestaltung: Christine Huth, Wiesbaden 


\section{Danksagungen}

Diese Untersuchung ist als Doktorarbeit an der Universität Hamburg entstanden. Für die Betreuung der Dissertation danke ich Prof. Dr. Kleinsteuber vom Institut für Politische Wissenschaft der Universität Hamburg, der mein Interesse an Medienpolitik und USA-Forschung geweckt hat. Bei ihm fand ich stets Unterstützung, nicht nur bei der Konzeption und Realisierung dieser Studie, sondern auch in früheren Phasen meines Studiums. Prof. Dr. Roß vom Institut für Journalistik der Universität Hamburg bin ich dafür verbunden, daß er die Aufgabe des Zweitgutachters übernommen hat.

Ein besonderer Dank geht an Dr. Renate Kosuch. Sie hat die Entstehung dieser Arbeit in allen Phasen engagiert begleitet. Ihre Fragen und Anregungen waren für mich eine große Hilfe. Für Korrekturhinweise danke ich außerdem Barbara Thomaß, meiner Kollegin an der Arbeitsstelle Medien und Politik der Universität Hamburg. Martin Hagen hat mich kompetent bei der Erstellung des Typoskripts unterstützt.

Die Idee, die organisatorischen Strukturen des Investigative Reporting zu analysieren, geht zurück auf eine Hospitanz, die ich 1989 beim Center for Investigative Reporting in San Francisco absolviert habe. Für die finanzielle Förderung dieses USA-Aufenthaltes bin ich dem Zentrum für Nordamerika-Forschung der Johann Wolfgang Goethe-Universität Frankfurt zu Dank verpflichtet. Durch die Gewährung eines Promotionsstipendiums hatte auch das Evangelische Studienwerk e.V. in Villigst wesentlichen Anteil daran, daß die hier vorliegende Untersuchung realisiert werden konnte.

Abschließend danke ich allen Gesprächspartnern in den USA, die sich bereitwillig für Interviews zur Verfügung gestellt und mir umfangreiches Material zugänglich gemacht haben. Durch ihre Offenheit und Kooperationsbereitschaft haben sie mir ermöglicht, einen praxisnahen Einblick in ihre Arbeit zu gewinnen. 


\section{Inhalt}

Verzeichnis der Abbildungen und Tabellen ............................................... 12

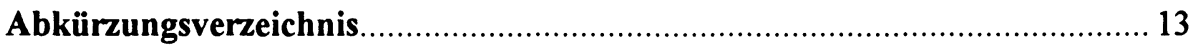

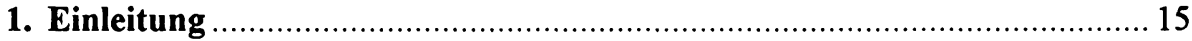

1.1 Stellenwert des Investigative Reporting im US-Journalismus ................... 17

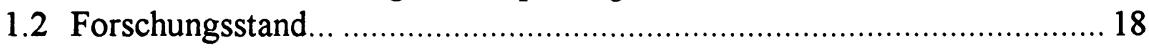

1.3 Fragestellung: Struktureller statt individueller Ansatz ............................22

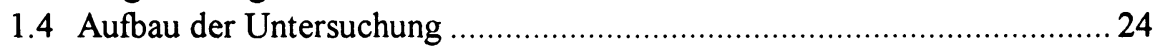

2. Einführung: Investigative Reporting als eigenständige Form

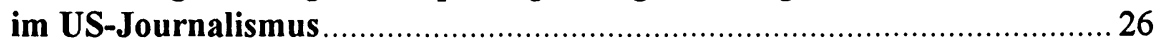

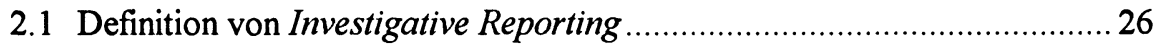

2.1.1 Ursachen der Begriffsunsicherheit............................................. 26

2.1.2 Definitionskonsens innerhalb der Investigative Reporters and

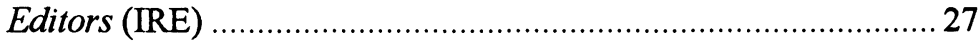

2.1.3 Betonung des normativen Gehalts: Investigative Reporting als journalism of outrage ................................................................... 30

2.1.4 Fazit: Arbeitsdefinition von Investigative Reporting ...................... 32

2.2 Abgrenzung von anderen Berichterstattungsmustern ................................ 35

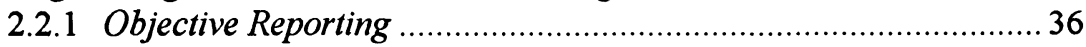

2.2.1.1 Vergleich zum Investigative Reporting …....................... 39

2.2.1.2 Exkurs: Ursachen einer deutschen Fehlinterpretation ....... 40

2.2.1.3 Muckraking als subjektivere Ausprägung des Investigative Reporting ................................................. 43

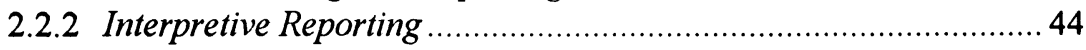

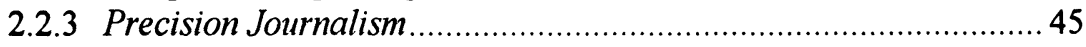

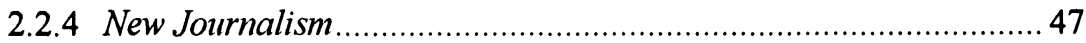

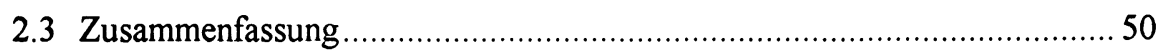

3. Methodische Vorüberlegungen für die Untersuchung .............................53

3.1 Normative Perspektive: Machtkontrolle durch Medien ............................ 54

3.1.1 Sichtweisen in den USA und in der Bundesrepublik ........................ 54

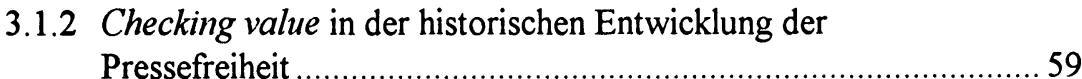

3.1.3 Interpretationen durch den Supreme Court..................................61

3.1.4 Watchdog role im Selbstverständnis der Journalisten...................... 63 
3.2 Strukturelle Perspektive und analytischer Rahmen: Bestimmungsfaktoren journalistischen Handelns

3.2.1 Von Weischenbergs Modell der Kontexte des Journalismus zu einem Modell der Faktoren, die Investigative Reporting beeinflussen 66

3.2.2 Das Konzept der politischen Kultur ........................................... 71

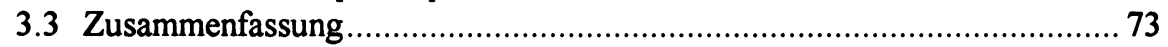

4. Historische Entwicklung des Investigative Reporting ................................75

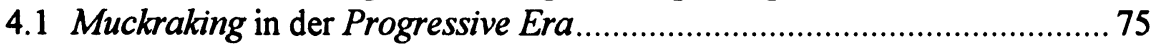

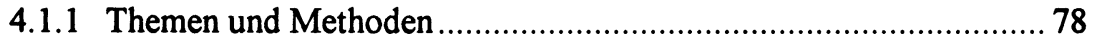

4.1.2 Verbindungen zum Progressive Movement ................................. 81

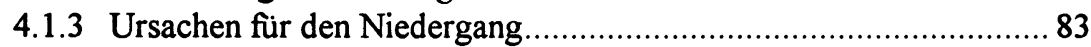

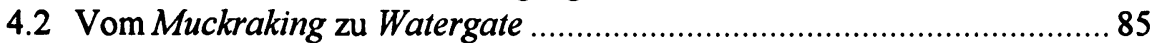

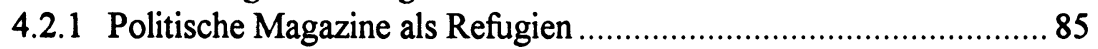

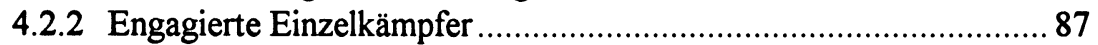

4.2.3 Lokalzeitungen und die Pionierrolle der Alternativpresse ............... 88

4.3 Zusammenfassende Schlußfolgerungen................................................. 90

5. Rahmenbedingungen für Investigative Reporting .......................................93

5.1 Politisch-gesellschaftliche Ebene: Politische Kultur.................................. 93

5.1.1 Nationalstolz und amerikanischer Grundkonsens.......................... 93

5.1.2 Skepsis gegenüber staatlicher Zentralgewalt................................ 96

5.1.3 Basisorientierung und Tradition der Selbsthilfe .............................99

5.1.4 Schlußfolgerungen für Investigative Reporting .......................... 101

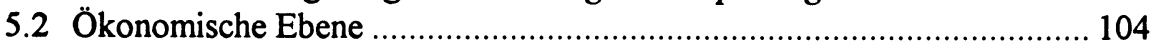

5.2.1 Kommerzialität als Grundzug des US-Mediensystems ................. 105

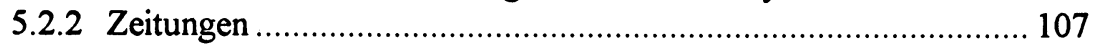

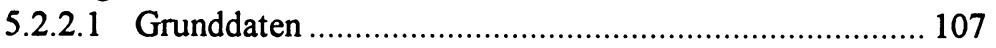

5.2.2.2 Konzentrationsprozeß und Rentabilität ........................ 109

5.2.2.3 Zurückgehende Leserbindung ..................................... 112

5.2.2.4 Neuer Management-Stil.................................................. 114

5.2.2.5 Schlußfolgerungen für Investigative Reporting ............. 118

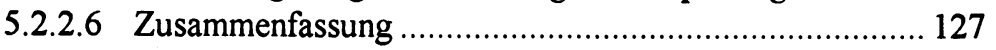

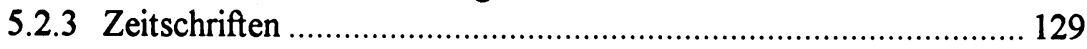

5.2.3.1 Trend zur Spezialisierung ......................................... 130

5.2.3.2 Nachrichtenmagazine unter Druck ................................ 131

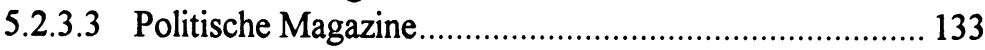

5.2.3.4 Zusammenfassende Schlußfolgerungen für

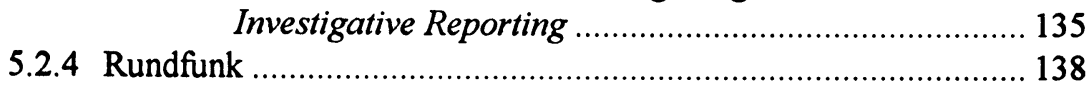

5.2.4.1 Grundstruktur des Rundfunksystems .......................... 138

5.2.4.2 Networks unter Konkurrenzdruck ................................ 142

5.2.4.3 Nicht-kommerzieller Rundfunk in der Nische............... 147 
5.2.4.4 Schlußfolgerungen für Investigative Reporting .............. 151

5.2.4.5 Zusammenfassung ..................................................... 158

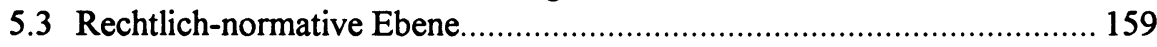

5.3.1 Informationsanspruch der Journalisten ..................................... 161

5.3.1.1 Open meeting laws und Freedom of Information Act ..... 161

5.3.1.2 Journalistischer Informationsanspruch vs.

Schutzrechte Einzelner ............................................... 164

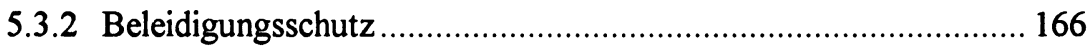

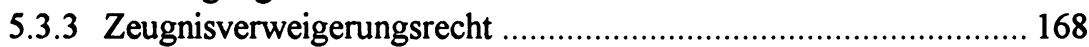

5.3.5 Schlußfolgerungen fur Investigative Reporting ......................... 170

5.3.5.1 Access laws als Stütze des Investigative Reporting ......... 170

5.3.5.2 Gegenstrategien zur Informationsblockade ................... 172

5.3.5.3 Beleidigungsklagen: Prozeßkosten bewirken

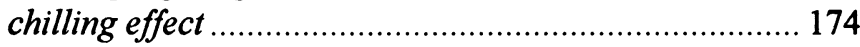

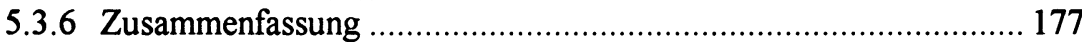

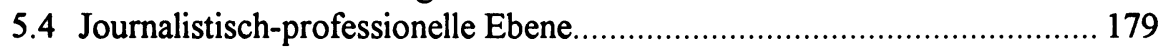

5.4.1 Zentrale Ergebnisse amerikanischer Kommunikatorstudien .......... 180

5.4.2 Rollenverständnis und ethische Standards ............................... 182

5.4.2.1 Bereitschaft zu kontroversen Recherchemethoden ......... 184

5.4.2.2 Ethik-Kodexe und andere Formen der professionellen Selbstkontrolle .................................................... 188

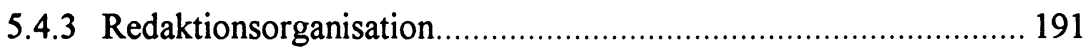

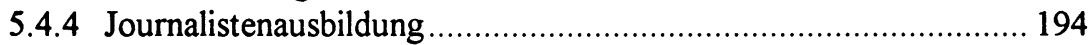

5.4.5 Schlußfolgerungen fur Investigative Reporting ......................... 197

5.4.5.1 Notwendigkeit der Ethik-Debatte ................................. 198

5.4.5.2 Investigative Reporting und öffentliche Glaub-

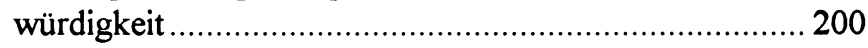

5.4.5.3 Investigative Reporting als eigenständiges

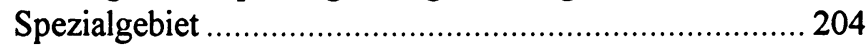

5.4.6 Exkurs: Computer-Assisted Reporting als Beispiel für eine neue journalistische Spezialisierung........................................... 206

5.4.6.1 Journalistische Nutzung von Datenbanken .................... 206

5.4.6.2 Verfügbarkeit und journalistische Verarbeitung von Daten aus der Verwaltung ........................................... 210

5.4.6.3 Computer-Assisted Reporting als neuer Kompetenzbereich im Investigative Reporting: Grenzen und

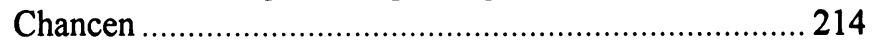

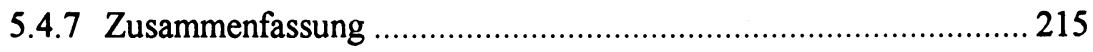

6. Organisatorische Struktur des Investigative Reporting ............................ 218

6.1 Verbreitung des Investigative Reporting nach Mediensparten................ 218

6.2 Auswahl der Untersuchungsobjekte für die Fallstudien ......................... 222

6.3 Investigative Reporters and Editors (IRE) als berufsständische

Organisation 
6.3.1 Entstehung und Organisationsweise.

6.3.2 Arizona Project: Katalysator in der Gründungsphase und

Beispiel für journalistische Kooperation.

6.3.3 Nicht-kommerzielle Vernetzung: Unterstützung durch

Spenden und Kooperation mit Universitäten ............................... 226

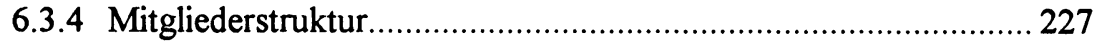

6.3.5 Leistungen für Mitglieder und Funktion im US-Journalismus ....... 229

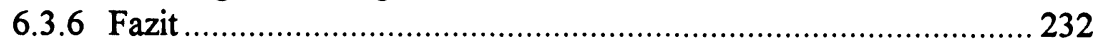

6.4 Investigative Reporting bei kommerziellen Medienorganisationen .......... 233

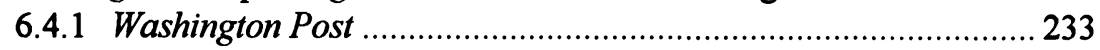

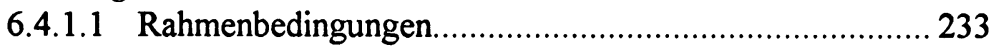

6.4.1.2 Stellenwert und Organisation von Investigative

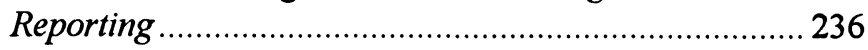

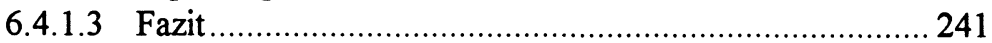

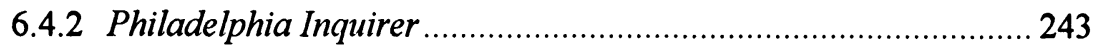

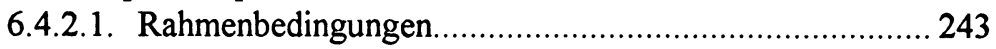

6.4.2.2. Stellenwert und Organisation von Investigative

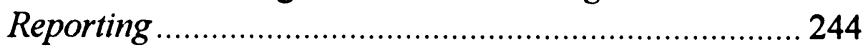

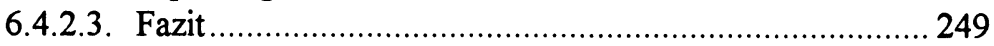

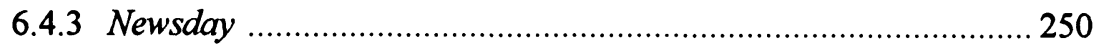

6.4.3.1 Rahmenbedingungen..................................................... 250

6.4.3.2 Stellenwert und Organisation von Investigative

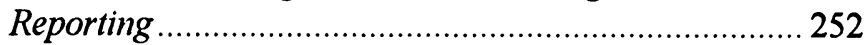

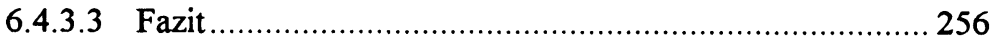

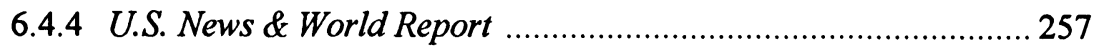

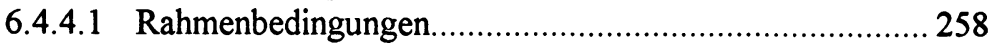

6.4.4.2 Stellenwert und Organisation von Investigative

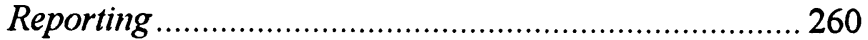

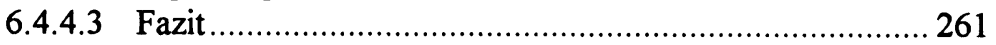

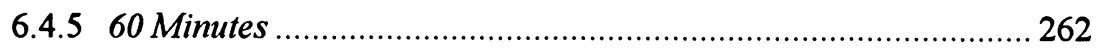

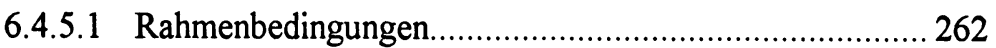

6.4.5.2 Stellenwert und Organisation von Investigative

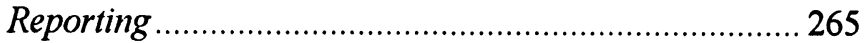

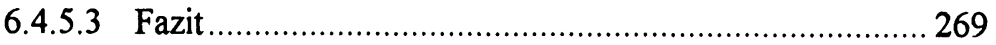

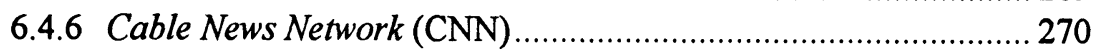

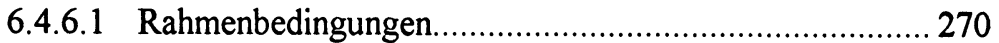

6.4.6.2 Stellenwert und Organisation von Investigative

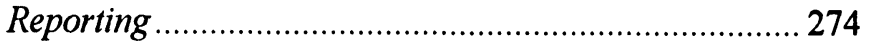

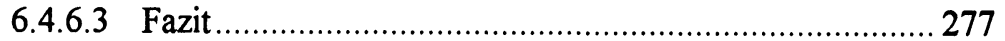

6.5 Investigative Reporting bei nicht-kommerziellen Organisationen ............. 278

6.5.1 Better Government Association ................................................ 278

6.5.1.1 Ziel und Entstehung der Organisation ........................... 278

6.5.1.2 Organisationsstruktur und Finanzierung ........................ 280

6.5.1.3 Arbeitsweise und Recherchebeispiele ............................ 281 


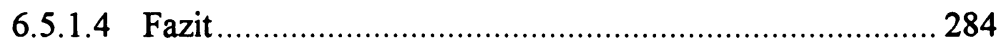

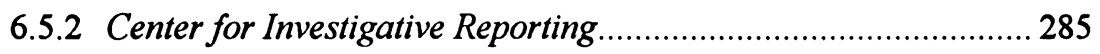

6.5.2.1 Zielsetzung und Organisationsstruktur .......................... 285

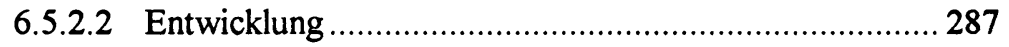

6.5.2.3 Nicht-kommerzielle Funktionen ...................................289

6.5.2.4 Sicherung der journalistischen Unabhängigkeit............... 291

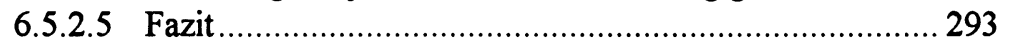

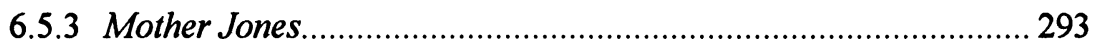

6.5.3.1 Zielsetzung und Organisationsstruktur........................... 294

6.5.3.2 Stellenwert von Investigative Reporting in der Entwicklung des Magazins..................................2295

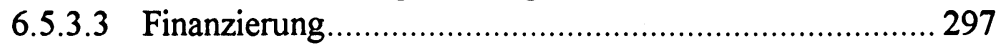

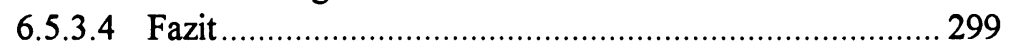

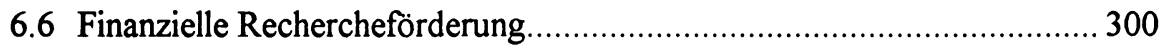

6.6.1 Fund for Investigative Journalism ............................................ 300

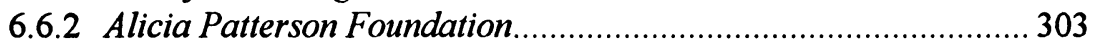

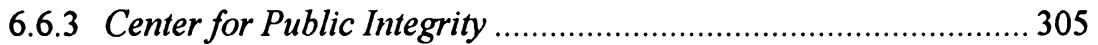

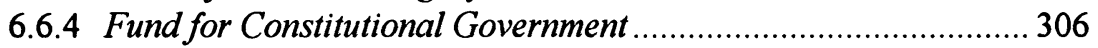

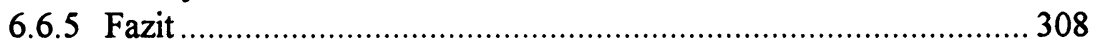

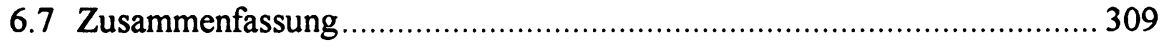

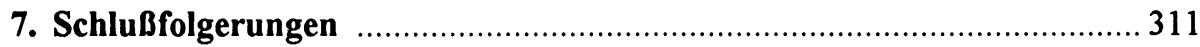

7.1 Strukturelle Voraussetzungen für Investigative Reporting:

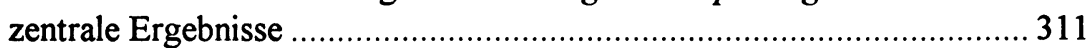

7.2 Investigative Reporting - ein Modell für die Bundesrepublik? .................. 316

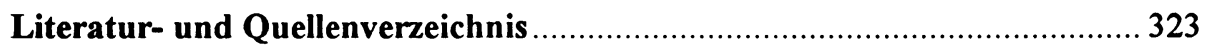

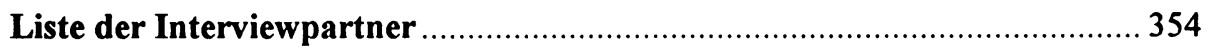

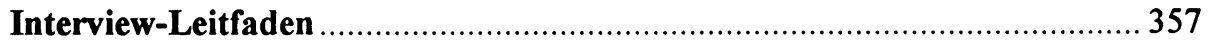


Abb. 1: Berichterstattungsmuster im US-Journalismus ..................................... 37

Abb. 2: Weischenbergs Modell der Kontexte des Journalismus........................67

Abb. 3: Modell der Faktoren, die Investigative Reporting beeinflussen............ 69

Abb. 4: Durchschnittliche Programmzahl, die in den TV-Haushalten

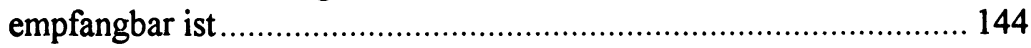

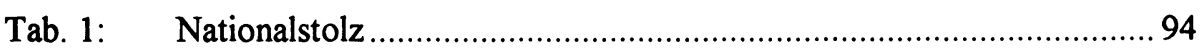

Tab. 2: $\quad$ Einschätzung der Ehrlichkeit bestimmter Berufsgruppen.....................98

Tab. 3: Die zehn auflagenstärksten Tageszeitungen der USA....................... 108

Tab. 4: Anzahl der redaktionell selbständigen Tageszeitungen nach

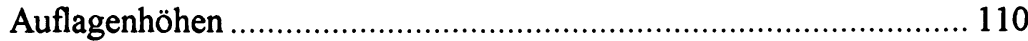

Tab. 5: Die 15 größten Zeitungsketten der USA ............................................ 111

Tab. 6: Verteilung der Werbeeinnahmen auf die Werbeträger ....................... 114

Tab. 7: Die zwanzig auflagenstärksten Zeitschriften der USA ...................... 131

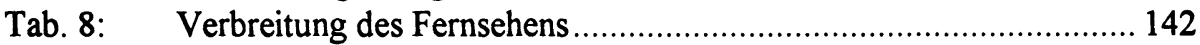

Tab. 9: Journalistisches Rollenverständnis ................................................ 183

Tab. 10: Legitimität umstrittener Recherchemethoden in den USA und in

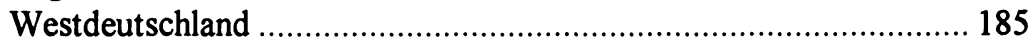

Tab. 11: Informationsquellen fur den letzten Bericht...................................... 193

Tab. 12: Bedeutung, die Investigative Reporting von der Bevölkerung

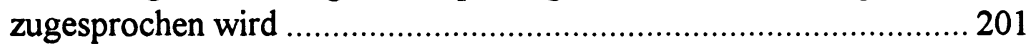

Tab. 13: Zustimmung zu umstrittenen Recherchemethoden............................ 202

Tab. 14: Verbreitung des Investigative Reporting bei Zeitungen und

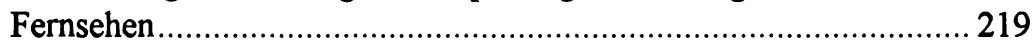

Tab. 15: Freistellung von Journalisten für Investigative Reporting ................. 221

Tab. 16: Arbeitsschwerpunkte der IRE-Mitglieder....................................... 228

Tab. 17: Einkommensquellen des Center for Investigative Reporting............. 292 


\section{Abkürzungsverzeichnis}

$\begin{array}{ll}\text { ABC } & \text { American Broadcasting Company } \\ \text { APF } & \text { Alicia Patterson Foundation } \\ \text { APME } & \text { Associated Press Managing Editors } \\ \text { APR } & \text { American Public Radio } \\ \text { ASNE } & \text { American Society of Newspaper Editors } \\ \text { BGA } & \text { Better Government Association } \\ \text { CAR } & \text { Computer-Assisted Reporting } \\ \text { CBS } & \text { Columbia Broadcasting System } \\ \text { CIR } & \text { Center for Investigative Reporting } \\ \text { CNN } & \text { Cable News Network } \\ \text { CPB } & \text { Corporation for Public Broadcasting } \\ \text { CPI } & \text { Center for Public Integrity } \\ \text { FCC } & \text { Federal Communications Commission } \\ \text { FCG } & \text { Fund for Constitutional Government } \\ \text { FIJ } & \text { Fund for Investigative Journalism } \\ \text { FOIA } & \text { Freedom of Information Act } \\ \text { GAP } & \text { Government Accountability Project } \\ \text { IR } & \text { Investigative Reporting } \\ \text { IRE } & \text { Investigative Reporters and Editors } \\ \text { IRS } & \text { Internal Revenue Service } \\ \text { MICAR } & \text { Missouri Institute for Computer-Assisted Reporting } \\ \text { NBC } & \text { National Broadcasting Company } \\ \text { NICAR } & \text { National Institute for Computer-Assisted Reporting } \\ \text { NPR } & \text { National Public Radio } \\ \text { PBS } & \text { Public Broadcasting System } \\ \text { PILI } & \text { Public Interest Law Initiative } \\ \text { PRI } & \text { Public Radio International } \\ \text { SPJ } & \text { Society of Professional Journalists } \\ \text { TBS } & \text { Turner Broadcasting System } \\ & \end{array}$

